\section{МЕХАНИЗМ НЕОИНДУСТРИАЛИЗАЦИИ: МЕТОДОЛОГИЯ ОБОСНОВАНИЯ}

\author{
И. А. Сушкова \\ кандидат экономических наук, доцент кафедры \\ экономики и финансового права, \\ Институт социального образования (филиал) Российского \\ государственного социального университета в г. Саратове \\ E-mail: irinasushkova60@mail.ru
}

Введение. Механизм осуществления неоиндустриализации экономики - предмет исследования экономической науки. В силу не столь продолжительного времени исследования проблем неоиндустриализации и сложности современной экономической ситуации, изменений законодательства, становления новой экономической политики серьезного изучения требует проблема создания эффективного механизма осуществления процесса неоиндустриализации российской экономики, а также выделения и задействования факторов его использования. Теоретический анализ. В статье исследуется содержание механизма осуществления неоиндустриализации экономики. Оно может быть построено на сформировавшихся в экономической науке методологических подходах к анализу хозяйственного механизма и с учетом методологии анализа процесса неоиндустриализации. Обсуждение результатов. Результатом разработанной автором проблемы механизма неоиндустриализации стало толкование понятия механизма неоиндустриализации, выделение его составных элементов, раскрытие конкретных результатов их взаимодействия, обоснование сущности и его общественного предназначения, проанализирована роль данного механизма в формировании технотронных произво-

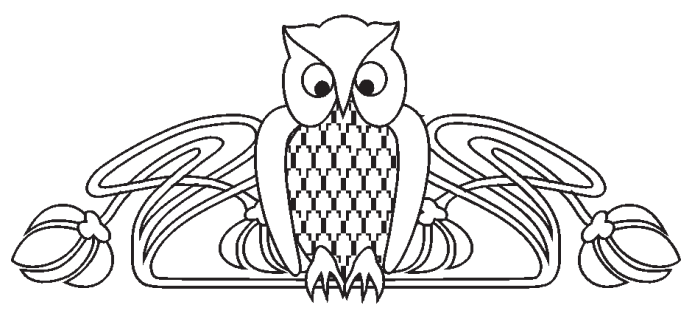

дительных сил и в переходе российской экономики к новому технологическому укладу.

Ключевые слова: неоиндустриализация, механизм неоиндустриализации, инновационное воспроизводство, хозяйственный механизм, новый технологический уклад, технотронные производительные силы, экономическая система.

\section{Введение}

В экономической литературе есть обоснование процесса неоиндустриализации через интеграцию предприятий различных отраслей, создание межотраслевых корпораций интегрированного типа, когда предприятия, входящие в их структуру, получают реальный доступ к современным промышленным и финансовым технологиям. Не менее интересными являются объяснения неоиндустриализации экономики через активизацию инвестиционной и инновационной деятельности на основе опережающего развития нового технологического уклада. При 
этом исследовались взаимодействия между государством, хозяйствующими и институциональными субъектами, а механизм осуществления рассматривался лишь в плане участия государства в регулировании этих процессов, в поиске мер и стимулов их активизации.

Даже самый общий анализ имеющейся литературы по неоиндустриализации позволяет сделать вывод о том, что вопрос о механизме ее осуществления требует дополнительного уточнения ряда проблем, серьезного обоснования и исследования. Пока не ясно, как будет решаться вопрос с собственностью, хотя стало очевидным, что при существующих отношениях собственности о прогрессивной трансформации экономической системы говорить не приходится. Не обозначили себя и не стали реальностью интегрированные цепочки производства наукоемкой продукции конечного спроса с высокой добавленной стоимостью. Не выработан алгоритм перехода на неоиндустриальный этап развития. Требуются обоснования задействования стимулов и мотиваций инновационной деятельности участников процесса неоиндустриализации, взаимодействия и направлений действия факторов экономического роста в направлении развития неоиндустриальных технологий.

\section{Теоретический анализ}

Мы будем исходить из того, что неоиндустриализация - это сложный экономический процесс, поэтому и реализация этого процесса совершается на основе механизма, представляющего собой сложное экономическое явление. Как экономическое явление механизм неоиндустриализации - это постоянно действующий механизм во времени и пространстве. Через него и благодаря ему государство направляет процессы новой индустриализации и создает условия для более эффективного их осуществления.

Этот механизм выступает элементом хозяйственного механизма, а потому реализуется он в ходе хозяйственной деятельности государственных институтов и хозяйствующих субъектов. Кроме того, механизм неоиндустриализации реализуется как механизм, обеспечивающий воспроизводство производительных сил и производственных отношений на новом, более высоком уровне развития. Поэтому анализ механизма неоиндустриализации целесообразнее вести на основе критерия хозяйственной и воспроизводственной целесообразности [1].

«Хозяйственность» и «воспроизводственность» этому механизму придает ряд обстоятельств:

- первое обстоятельство состоит в том, что участники процесса неоиндустриализации реализуют свои функции в социально-экономической системе как хозяйствующие субъекты, осуществляющие процесс воспроизводства основного и оборотного капитала;

- второе - механизм неоиндустриализации есть механизм реализации хозяйственных решений субъектов, связанных с обновлением и модернизацией производства и направленных на достижение хозяйственных целей (производство новой продукции на основе новых технологий, что включает соответствующее потребление ресурсов и получение результатов);

- третье - механизм неоиндустриализации есть организация формирования и воспроизведения производительных сил общества нового технологического уклада и стимулирование их эффективного использования, что в принципе составляет внутренний момент хозяйствования, его содержательный смысл;

- четвертое - механизм неоиндустриализации есть механизм формирующий, организующий, управляющий, воспроизводящий систему, в которой «господствуют» знания, инновации, интеллектуальный труд;

- пятое - механизм неоиндустриализации есть элемент хозяйственного механизма, через который реализуется закон вертикальной интеграции, согласно которому извлечение прибыли допускается из конечного обрабатывающего производства.

В силу этих обстоятельств механизм неоиндустриализации является по своей основе и характеру хозяйственным, а по логике действия воспроизводственным, потому функционирует в структуре хозяйственного механизма как его элемент, а по сути, становится главным элементом хозяйственного механизма, подчиняющим себе функционирование всех других его составных частей. Можно допустить, что хозяйственный механизм социально-экономической системы в силу надобности постоянного поддержания процесса формирования производительных сил на уровне нового знания и новых технических и технологических открытий может и должен приобретать качества и свойства механизма, поддерживающего индустриализацию постоянно, то есть механизма новой индустриализации.

Основываясь на этих общих выводах, можно утверждать, что механизм неоиндустриализации как элемент хозяйственного механизма принадлежит экономической системе, ею порожден на этапе инновационного скачка в новый технологический уклад и ею определяется.

Механизм неоиндустриализации предстает как взаимодействие организационно-хозяйственных форм, методов управления инновационной деятельностью, инструментов стимулирования и использования ее результатов, условий принятия инновационных решений. Благодаря этому взаимодействию данный механизм обеспечивает переход к новому типу экономического роста 
и имеет следующую поэлементную структуру: инструменты стимулирования процесса неоиндустриализации; методы управления процессом неоиндустриализации; новые организационнохозяйственные формы; заинтересованность и ответственность субъектов за процессы неоиндустриализации. Перечисленные элементы механизма неоиндустриализации сами имеют содержательные составляющие, что в конечном итоге способствует появлению новых элементов механизма, усложняет их взаимодействие, усиливает синергетический эффект этого взаимодействия.

Накопление знаний и трудового опыта всегда вело к инновационным переменам в социально-экономическом обустройстве общества. Вместе с этим менялось и восприятие этих перемен. Постепенно складывалась экономическая философия, опирающаяся на принцип применения научных новшеств, их внедрение в конкретную практику в качестве инструмента улучшения условий и повышения качества жизни населения, роста производительности труда и ресурсоотдачи. Приложение такой философии к экономике, стоящей перед выбором пути развития, фактически послужило выработке нового государственного курса - курса на формирование и использование наиболее действенных элементов механизма неоиндустриализации экономики.

Сущность механизма неоиндустриализации состоит во взаимодействии его элементов, которое предстает как адекватно осуществляемое соотношение функционирующих экономических отношений и устанавливаемых государственными институтами норм и правил (форм экономических связей) их реализации, способное обеспечить создание добавленной стоимости, достаточной для постоянного обновления наукоемкого оборудования и расширенного его воспроизводства, функционирование оптимальных форм организации ее движения и присвоения.

От того, как этот механизм функционирует, насколько он реален и результативен, зависит состояние экономической системы, ее способность к эффективному техническому и технологическому обновлению. Механизм новой индустриализации есть механизм, посредством которого:

- создаются условия для полной автоматизации и компьютеризации производительных сил отечественного народного хозяйства, с интенсивным замещением трудоемкого производства наукоемким и машиноемким;

- обеспечивается реализация оптимального способа формирования новой экономической системы, гарантирующей решение масштабных задач неоиндустриального прорыва к высшей стадии развития.

Важнейшее предназначение механизма новой индустриализации состоит в том, что в процессе его функционирования совершается переход к новому технологическому укладу. Возникает феномен инновации, а вместе с ним и феномен конечной прибыли интегрированных межотраслевых комплексов. Интегрированные субъекты осуществляют свою деятельность (интегрированное воспроизводство за счет новых открытий, изобретений, технологий, творческого труда, инновационного капитала) ради получения результата - большей прибыли из конечного обрабатывающего производства. Самовозрастание интегрированного капитала становится уже не просто вложением его в дело, а авансированием в добывающие, промежуточные и обрабатывающие производства в пользу ожидаемого дохода. Такое движение интегрированного капитала обеспечивает инновационное воспроизводство, нацеленное на конечный результат.

На механизм неоиндустриализации выпадает главная «обязанность» организовать хозяйственную систему, обеспечить ресурсами расширенное воспроизводство материальных благ и услуг, да и сама система выступает его функцией. Стало быть, именно функционирующий механизм неоиндустриализации способствует формированию составляющих ее необходимых процессов и созданию между ними взаимозависимостей. Такой действенный механизм организует конкретные процессы, через которые в обществе и совершается формирование новых производств, их структуризация, процессы приспособления, изменчивости и развития, созидания и разрушения, противоречия.

К таким процессам можно отнести:

- формирующееся многообразие технологических укладов;

- активизация инвестиционной и инновационной деятельности;

- формирование новых производственнотехнологических комплексов;

- создание системы стратегического планирования;

- активная промышленная политика, стимулирующая «точки роста»;

- поддержание макроэкономических условий устойчивого развития и т.д.

Практическое действие механизма неоиндустриализации есть всестороннее использование главного предназначения государства: планирования, управления, регулирования. Осуществляя планирование, управление, регулирование, государство создает благоприятные экономические условия для инновационной деятельности через налоговую и амортизационную политику; через систему подготовки и переподготовки кадров; через научно-технологические комплексы и технопарки; через совершенствование залогового кредитования субъектов, финансового лизинга движимого 
и недвижимого имущества; через проведение переоценки основных фондов в соответствие с темпами инфляции; через создание и развитие сети информационно-аналитических центров, осуществляющих проведение рейтингов и рейтинговых оценок субъектов инновационной деятельности, и др.

Адекватность и эффективность осуществления механизма определяется тем, насколько предпринимаемые меры со стороны государства соответствуют отношениям, в которые оно, в лице специальных экономических и правовых институтов, как хозяйствующий субъект вступает (производственные отношения и отношения продуктообмена, отношения воздействия и взаимодействия с инновационными субъектами).

В структуру механизма неоиндустриализации входят следующие группы факторов:

- экономически предопределяющих заинтересованность хозяйствующих субъектов в использовании конкурентных рычагов в максимизации прибыли, способов снижения издержек и повышения отдачи от используемых ресурсов, способов обновления основных фондов и увеличения оборотных средств, интеграции и специализации; финансовые, денежно-кредитные, инвестиционные, налоговые факторы мотивации и стимулирования инновационной деятельности хозяйственных субъектов;

- политически обеспечивающих системную инновационную направленность экономики на установление запретов на внеэкономический передел собственности в пользу компрадорского и неразрывно с ним связанного зарубежного капитала; введение в действие налогового механизма национализации значительной части природной ренты, прежде целиком присваиваемой компрадорским капиталом; введение законодательных норм, регулирующих экспорт топливно-энергетических ресурсов в общегосударственных интересах; установление правил формирования валютных резервов страны; регулирование государственной части внешнего долга; обеспечение суверенитета и экономической самостоятельности России; формирование каналов повышения внутренней составляющей совокупного спроса через государственные заказы и закупки, а также национальные проекты;

- социально преобразующих общий климат в стране путем повышения уровня жизни населения, обеспечения работой, достойной заработной платой, социальными услугами, комфортным жильем, доступностью медицинских услуг, отдыха, объектов культуры, туризма, развлечений, гарантии безопасности и правовой защиты.

Одновременное скоординированное использование этих факторов позволит вывести отечественное хозяйство на новый качественный уровень развития, но лишь при условии:
- соблюдения баланса компаний различных форм собственности, конкурирующих между собой;

- обеспечения равного доступа к ресурсам;

- наличия налоговой системы: по форме внятной, по сути - стимулирующей, по способу осуществления - с полученной прибыли, по времени действия - стабильной на протяжении длительного срока;

- установление реального адекватного уровню решаемых в экономике проблем государственного надзора на финансовом рынке.

Именно одновременное, оптимально скоординированное использование выделенных выше факторов и условий их действия обеспечит сочетание интересов государства, бизнеса и населения в реализации индустриальных перемен, что, в свою очередь, явится обязательным условием экономической надежности, независимости и безопасности национальной экономики. Такие последствия могут быть обеспечены в силу того, что действие данных факторов формирует:

1) возможности для разработки обоснованных стратегических инновационных программ социально-экономического развития страны, их политическую поддержку со стороны государства, прозрачность целей и действий бизнеса, реализации его социальной ответственности и ответственности за эффективное использование национальных ресурсов;

2) мотивы актуализации научно-технического потенциала страны в обеспечении его возможностей в создании и освоении научно-технических разработок, отвечающих мировому уровню;

3) различные адекватные формы государственной поддержки научной, научно-технической и инновационной деятельности;

4) благоприятные возможности достижения согласованности экономических интересов субъектов: предпринимательских структур, государственных институтов, всех групп населения, крупного и мелкого бизнеса.

В рамах теоретического обоснования установления оптимальности взаимодействия экономических, политических и социальных факторов особое место занимает выбор методов их реализации. Вопрос этот один из самых непростых.

Сейчас в ходе обсуждения проблем выхода российской экономики из системного кризиса стало обычным, в определенной мере справедливым, отвергать монетарные регуляторы. В качестве довода и оправдания тому считается, что неотложные задачи неоиндустриального развития требуют включения механизма планово-централизованной концентрации реальных ресурсов на приоритетных направлениях структурно-инновационного маневра и прорыва [2, c. 9]. Вполне разумно и оправданно поддержать эту идею. Но поскольку такие предложения 
звучат постоянно и критика монетарных регуляторов звучит все настойчивее, то возникает опасение отказа от денежных и финансовых регуляторов настолько, что мы сможем впасть в другую крайность. И не улучшить ситуацию, а еще более углубить разрушительные процессы, свойственные российской экономике, которые и так принесли слишком много бед.

Очевидно, что вопрос о планировании в условиях рынка все настойчивее выходит на повестку дня. В этой связи приходится пересматривать и роль государства в происходящих переменах, в руководстве экономикой.

Достижение согласованности и оптимальности взаимодействия экономических, политических и социальных факторов в механизме реализации новой модели экономического развития возможно при безусловном выполнении следующих принципов: стратегического ориентирования в развитии экономической системы; оптимального согласования и использования плановых и рыночных начал в развитии экономики; финансовой поддержки научно-технической и инновационной деятельности; интеграции науки, образования и производства; обязательного включения в программы стратегического развития мероприятий по проведению НИОКР, освоению научных результатов и организации собственного производства дефицитных и импортозамещающих материалов и оборудования; достижения комплементарности и поэтапности изменения интересов предпринимательских структур и государственных институтов; открытости информационных потоков в целях и перспективах власти и бизнеса; обеспечения условий достойного существования и всестороннего развития всех членов общества.

Соблюдение данных принципов в процессе установления оптимального соотношения экономических, политических и социальных факторов в модели неоиндустриального развития - условие реализации функций государства в экономической системе, функций экономики в направлении обеспечения инновационных перемен, функций социальной сферы в общих социально-экономических преобразованиях страны.

При обеспечении этих условий и направленных усилий политической и экономической власти, социума российского общества может быть достигнуто оптимальное сочетание факторов: политически обеспечивающих системную инновационную направленность экономики; экономически предопределяющих свободу выбора хозяйствующих субъектов при определении способов ее достижения; формирующих новый, адекватный социокультурный слой общества, завершающий определение общей инновационно-индустриальной направленности развития государства.
Проблема оптимального сочетания данных факторов в модели экономического развития неразрывно связана с проблемой использования факторов повышения эффективности производства и обеспечения его неоиндустриализации. Дело в том, что при всем разнообразии форм и направлений роста эффективности конечной целью и итогом общественного воспроизводства остается достижение иного качества производства как основы создания условий наиболее полного удовлетворения потребностей каждого члена общества, реализации творческого потенциала каждого коллектива.

Поэтому оптимальное соотношение задействованных факторов регулируемого обеспечения инновационного развития хозяйства, экономической свободы хозяйствующих субъектов и возможностей формирования социокультурного слоя в общей структуре государственного образования может рассматриваться как условие реализации закона повышения эффективности воспроизводственного процесса, что предполагает ускорение оборачиваемости капитала с возвышающейся отдачей в ходе оптимальной смены его стадий. Действие закона повышения эффективности воспроизводственного процесса обусловливает возможность достижения желаемого результата:

- экономика приобретет инновационно-инвестиционную направленность;

- субъекты хозяйства примут новый технико-технологический облик;

- «невидимая рука» частного интереса станет эффективно работать на интерес общественный - обеспечение инновационной трансформации социально-экономической системы России.

\section{Обсуждение результатов}

Механизм неоиндустриализации - это механизм, который в ходе своего функционирования обеспечивает такое взаимодействие рыночного механизма и механизма государственного регулирования, при котором появляются условия для инновационной деятельности хозяйствующего субъекта, обеспечивается простор для функционирования всех форм хозяйствования, результативно действуют инструменты управления и стимулирования инноваций, а субъекты имеют возможность реализовать свои интересы. Иными словами, механизм неоиндустриализации это механизм инновационно ориентированного развития социально-экономической системы.

Формируется механизм неоиндустриализации в ответ на запрос самой экономической системы постепенно, по мере ее развития. Причем формируется он как сложное системное образование, включающее такие структурные элементы, которые в своей совокупности способны обе- 
спечить развитие системы, его порождающей. Стало быть, механизм неоиндустриализации формируется вместе с трансформирующейся системой, в которой меняется положение социальных групп, их соотношение, взаимодействие с властью, степень общественной активности, уровень ответственности за страну, за состояние производства, за общественный климат, за уровень зарплаты, за воспитание и образование подрастающего поколения, за подготовку специалистов.

Специфичность этого механизма определяется целевыми установками индустриализации экономики, которые имеют общую направленность на получение добавленной стоимости за счет производства высококачественной, высокотехнологичной продукции - продукции глубокой технико-технологической переработки имеющихся ресурсов. Именно эта направленность и определяет поэлементную структуру механизма и их взаимодействие.
Стало быть, механизм осуществления новой индустриализации экономики - сложное системное образование, включающее факторы, инструменты, принципы, стимулы и движущие силы создания новых и обновления действующих производительных сил общества.

Он обеспечивает инновационное воспроизводство - воспроизводство интегрированного межотраслевого комплекса, расширяющего свои масштабы, экономно использующего ресурсы, заботящегося о сокращении затрат в своих интересах и заинтересованного в проникновении в другие сферы.

\section{Список литературы}

1. Сушкова И. А., Калашников И. Б. Новая индустриализация российской экономики : идеи, практика, обоснование. Саратов, 2014. 234 с.

2. Кучуков Р. Государственный сектор и его роль в стратегии - 2020 // Экономист. 2012. № 9. С. 3-14.

\title{
Mechanism of Neoindustrialization: Methodology of Justification
}

\author{
I. A. Sushkova \\ Candidate of Economic Sciences, Associate Professor, \\ Department of Economy and Financial Law, \\ Institute of Social Education (branch) \\ of Russian State Social University in Saratov, \\ 4, Fabrichnaja str., Saratov, 410019 Russia \\ E-mail: irinasushkova60@mail.ru
}

Introduction. The mechanism of implementation of neoindustrialization of economy is an object of the research of economic science. Due to the short time the researches of the problems of neoindustrialization and complexity of modern economic situation, the changes of the legislation, the formation of new economic policy the problem of creation of the effective mechanism of implementation of the process of neoindustrialization of Russian economy and also the allocation and the involvement of the factors of its use requires serious study. Theoretical analysis. In the article the content of the mechanism of implementation of neoindustrialization of economy is investigated. It can be built on the methodological approaches to the analysis of economic mechanism, created in economic science, and taking into account the methodology of the analysis of process of neoindustrialization. Discussion of results. The result of the problem of the mechanism of neoindustrialization developed by the author was the interpretation of concept of the mechanism of neoindustrialization, the allocation of its components, the disclosure of concrete results of their interaction, the justification of the essence and its public mission, the role of this mechanism in the formation of texnotronical productive forces and in the transition of Russian economy to a new technological way is analysed.

Key words: neoindustrialization, mechanism of neoindustrialization, innovative reproduction, economic mechanism, new technological way, texnotronical productive forces, economic system.

\section{References}

1. Sushkova I. A., Kalashnikov I. B. Novaja industrializacija rossijskoj jekonomiki: idei, praktika, obosnovanie [The new industrialization of the Russian economy: ideas, practice, rationale]. Saratov, 2014. $234 \mathrm{p}$.

2. Kuchukov R. Gosudarstvennyj sektor i ego rol' v strategii - 2020 [The public sector and its role in the strategy-2020]. Jekonomist, 2012, no. 9, pp. 3-14. 